\title{
NUEVA AGENDA PARA LAS PRÁCTICAS EVALUATIVAS
}

\author{
Graciela Iturrioz * \\ Universidad Nacional de la Patagonia San Juan Bosco, Argentina \\ mgiturrioz@gmail.com
}

Recibido: 9/06/2020 - Aceptado: 19/11/2020

\section{Resumen}

La evaluación de los aprendizajes es siempre un tema complejo, que por el lugar que ocupa en los sistemas educativos adquiere siempre matices controversiales. Del contexto de continuidad pedagógica actual, emergen nudos críticos que interesa recrear en este trabajo en torno a los problemas pendientes del pasado como hacia los legados que reportará al debate en el campo, a partir de anticipaciones que resultan de conversaciones docentes.

Palabras claves: Evaluación - Aprendizaje - Entornos - Prácticas.

\section{RESERVOIR OF NEW ASSESSMENT PRACTICES}

\begin{abstract}
The evaluation of learning is always a complex issue, which, due to its place in educational systems, always acquires controversial nuances. From the context of current pedagogical continuity, critical nodes emerge that are interesting to recreate in this work around the pending problems of the past as well as the legacies that it will report to the debate in the field, based on anticipations that result from teaching conversations.
\end{abstract}

Keywords: Assessment - Learning - Environments - Practices.

\section{Marco de análisis}

Con las escuelas cerradas físicamente por efecto de la suspensión de clases presenciales en el tiempo actual, la educación continuó fuera de sus muros, en el marco de la denominada continuidad pedagógica. De pronto, los docentes dejaron de enseñar en las aulas para hacerlo por medios electrónicos y con el uso de cuadernillos de estudio propuestos por el Estado, en algunos casos. Las familias pasaron a asumir responsabilidades nuevas, y los alumnos a acomodarse a esta nueva modalidad de escolarización fuera de las aulas y alejados de sus compañeros y amigos.

Lo cierto es que surgen y surgirán innumerables dilemas, interrogantes y problemas que los estudios que se emprendan ilustrarán. Para nuestro interés, y en el marco de un espacio de diálogo fecundo con los docentes que luego se describe, adelantamos algunos que se refieren al tema de la evaluación. No con el objetivo de hablar de la evaluación en y por la pandemia, sino de restaurar el debate sobre miradas ríspidas y proponer.

\footnotetext{
* Profesora y Licenciada en Ciencias de la Educación. Magister en Psicología cognitiva y aprendizaje escolar FLACSO Argentina. Doctora de la Universidad de Buenos Aires en Ciencias de la educación. Profesora regular de la Facultad de Humanidades y Ciencias Sociales de la Universidad Nacional de la Patagonia San Juan Bosco. Investigadora de la misma institución.
} 


\section{La experiencia de fuente}

La Resolución CFE N ${ }^{\circ} 363 / 20^{1}$ despertó un conjunto de preocupaciones en pleno desarrollo virtual de la educación, en docentes de escuelas primarias y secundarias, que se divulgó casi como un rayo. Condujo a entender que había promociones automáticas, sin instancia de evaluación formal, que los docentes recogieron con desasosiego e incertidumbre.

Casi de modo inmediato circuló la advertencia de los docentes, que indicaba que lo poco que avanzaban con los estudiantes se estaba perdiendo en virtud de que ya no habría calificación y el pase entre niveles sería automático. Como también debemos decir que esta percepción se solapó con otra que indicaba que se perdía el ciclo lectivo.

Dando curso a un sitio para conversar acerca de sus preocupaciones en torno a la evaluación y su confuso estado en el marco de la citada norma, organizamos un taller de educadores que reunió a un importante número de docentes para conversar sobre el tema, que se desempeñan en seis escuelas secundarias de la zona. Las sorpresas fueron grandes, los debates intensos y las anticipaciones de problemas vastas.

El espacio constituido resultó de un movimiento espontáneo por parte de directivos de las escuelas participantes de la ciudad de Comodoro Rivadavia, a la que luego se sumó una de la ciudad de Rawson, ambas de la Provincia del Chubut; sus inquietudes en torno a la evaluación surgieron disparadas no solo por las preocupaciones consecuentes de la novedad de la experiencia, sino también por la circulación de la Res. ME Chubut 71/20, la norma jurisdiccional que adoptó la Res. CFE 363/20 que suscitó diferentes planteos, preguntas e inquietudes respecto a las decisiones evaluativas que habría que tomar vista la proximidad del cierre del próximo trimestre. Por razones de extensión del artículo, se mencionarán a continuación algunos trazos del taller, a fin de asignar mayor contextualización a su posterior análisis.

Las preguntas docentes se centraron mayoritariamente en la asignación de calificaciones a los estudiantes como consecuencia del desarrollo del primer trimestre del año en curso sin referencias tangibles suficientes de sus actuaciones académicas. En algunos casos, contaban con las producciones resultantes de los cuadernillos de estudio que proveyó el Ministerio de Educación de la Nación o de las actividades que proporcionaban los docentes, aunque lo hacían parcialmente, fuera de tiempo y de las consignas dadas 0 nunca. Consideraban que se generarían situaciones injustas con quienes cumplían con la entrega y que se banalizaría la evaluación y acreditación en la contemplación de las situaciones personales.

Otras preocupaciones destacadas se expresaron respecto a la autoría de las producciones de los estudiantes, en tanto suponían que eran realizadas por terceros, o que el contenido provenía de fuentes de internet, sin mediación personal en la construcción de conclusiones. También existieron las voces que manifestaron que había desigualdad en el punto de partida, en tanto muchos estudiantes no contaban con conectividad suficiente para estudiar o no alcanzaba la provisión de material didáctico impreso comentado. A ello se sumó el importante alcance de la medida de fuerza docente en toda la provincia en virtud del atraso salarial, que generaba mayor tensión al panorama descripto.

Con base en las voces descriptas, el taller de educadores se estructuró sobre la base de preguntas y planteo de problemas, que atendieron a desarmar esa impenetrable capa de argumentaciones que se mostraba concentrada y consistente. Las preguntas fueron recurrentes a lo largo del taller y se centraron en el reconocimiento de los problemas efectivos que visualizaban en el contexto que describían y de otros aprendizajes que surgían en la vida de niños y jóvenes mientras transcurría la continuidad pedagógica. Ambas preguntas y problemas se entrecruzaban de manera recurrente, con el objetivo de desandar visiones muy consolidadas que entraban en conflicto cuando se trataba de pensar el valor de referencias empíricas de aprendizaje fuera de la escuela y de lo convencional, aunque asociadas sin duda alguna a ellos.

Las normativas indicadas, Res. CFE 363/20 y Res. MEChubut 71/20 representaron entonces, a la vez que un articulado de argumentaciones en apariencia válidas acerca del problema de evaluar fuera de los marcos y prácticas habituales, por lo que las intervenciones propuestas en el taller se centraron en la mirada analítica de sus consideraciones en línea con los problemas que expresaban los docentes.

En lo que viene se describen algunas entradas de este espacio, nuestra reflexión y propuestas acercadas, respecto de las prácticas evaluativas.

\section{Recuperar el legado}

Nada ha de ser igual al volver. Nos preguntamos entonces cómo recuperaremos el legado de lo vivido. A pesar de no acontecer la vuelta, los docentes advierten la emergencia de un nuevo escenario. Entendemos que habría un valioso legado a recuperar que requerirá un reconocimiento exhaustivo de los diferentes actores, cada uno en su perspectiva, y para lo cual la institución escolar deberá habilitar espacios que lo posibiliten.

\footnotetext{
${ }^{1}$ Esta normativa aprobó, atendiendo a los contextos y realidades jurisdiccionales, orientaciones para la evaluación en el marco de la continuidad pedagógica dada por efecto del cierre presencial de las escuelas en el ciclo lectivo 2020 . Entre ellas, se destaca el énfasis en la valoración de los logros de los estudiantes durante el acompañamiento, de modo de evitar decisiones excluyentes hacia quienes no pudieron acceder a la educación. El marco conceptual que la sustenta es la evaluación formativa, representada en el valor de proporcionar información a los estudiantes y sus familias por sobre la asignación de calificación numérica o conceptual, con consideración especial a la heterogeneidad.
} 
En esta recreación, importará recuperar, en primer lugar, las mediaciones que intervinieron en la enseñanza y en el aprendizaje, instrumentales y sociales. Baquero, (1997) cuando analiza la obra vygotskiana, explica que el centro del planteo del gran pensador ruso fue la actividad instrumental y la interacción social, y en la primera distinguió instrumentos que modifican externamente la actividad humana y signos que lo hacen internamente en la interiorización. Respecto de la interacción social, se trata de una experiencia constitutiva de la vida humana, sin la cual perderíamos nuestra condición. La teoría vygotskiana la sitúa esencial para el desarrollo de los procesos psicológicos superiores.

Si en el escenario de vuelta a la escuela, ese estudio de las mediaciones se hace mediante la evaluación, visualizamos un desafío enorme, porque se trata de mirar de manera precisa los instrumentos de evaluación que se emplearán. Si bien nos referiremos a ello a posteriori, es menester expresar el compromiso ineludible de construir instrumentos que miren la experiencia no solo desde las mediaciones escolares sino desde el enorme acervo de recursos que intervinieron en la vida de los estudiantes en su vida cotidiana.

Entendemos que el legado será doble; por un lado, el inventario de mediaciones y su análisis hacia el currículum, en términos del largo debate respecto de la tensión entre los saberes escolares y cotidianos, que en este escenario de situación se ha expresado con toda su fuerza; por otro, sobre la forma de evaluar, en términos de visualizar otra forma de construir instrumentos de evaluación y de abordar de manera analítica el problema de la calificación y construir propuestas.

En tal sentido, no se trata de pensar los efectos de la enseñanza mediada solo experiencialmente, sino de activar una vez más la necesidad de repensar el problema de los cambios culturales que la escuela necesita.

\section{Censar los entornos}

La recuperación del legado supondrá reconocer el infinito universo de experiencias que adolescentes y jóvenes transitaron en el estudio en casa. Será esencial reconocer esos entornos en los que se produjo el estudio que permita visualizar el repertorio de estrategias que emplearon para hacerlo, para ponerlo en valor y adscribirlo al quehacer presencial.

Una herramienta valiosa en esta travesía censal debiera ser justamente la evaluación, que fuera el motivo de la experiencia de encuentro en el taller en que aconteció el encuentro, y ello por dos razones. Primero, porque supondría hacer uso de una evaluación auténtica (Díaz Barriga, 2005) que remite a recrear dispositivos en modo artefactos culturales, que se acerquen a los aspectos importantes del entorno de los sujetos que se evalúan desde una mirada sociocultural. Adoptar este posicionamiento supondría generar consignas evaluativas que favorezcan justamente lo acontecido en esos entornos cotidianos en los que transcurrió la continuidad pedagógica. Y segundo, porque podría generar una legitimación de los saberes que se relevan. Numerosos estudios han develado que existe en los estudiantes una fuerte tendencia a estudiar para la evaluación, esto es, para los modos en que la perciben, pues así creen que serán calificados. Derek Rountree (en Camilloni, 2015) explica que orientan, organizan y deciden sobre la forma de estudiar en tanto indique el sistema de evaluación, lo que hace que el esfuerzo se ponga solo en lo que se va a preguntar; en otras palabras, los estudiantes saben que cuando un saber está en la prueba es porque importa, y legitima así ese saber. En este caso, su potencial legitimador podría actuar en el otro sentido, es decir, que cuando preguntemos sobre sus aprendizajes cotidianos relacionados con saberes escolares, estaremos generando una legitimación de esa intersección al interior del currículum escolar. Sería una sabrosa oportunidad, que posibilitaría una evaluación genuina en términos del análisis anterior.

Los docentes expresan que los estudiantes han adquirido habilidades diversas, que van desde competencias digitales hasta domésticas, y emocionales para la mejor convivencia y el estado de ánimo. Censarlas y adscribirlas al currículum escolar es una forma de dar curso y lugar a esa diversidad, que ya es parte de su identidad. $Y$ en esos nichos, distinguir tareas, contextos favorecedores y no tantos, estrategias valiosas, resultados logrados. Dicen por ejemplo que han construido hábitos de estudio, que les posibilitaron ordenar sus jornadas, y así emprender labores domésticas, recreativas e intelectuales. También que los ayudan en el dominio de las herramientas tecnológicas, en las videollamadas y en las herramientas del entorno virtual; relatan que extrañan la escuela como ámbito de encuentro con amistades e indican los esfuerzos que deben hacer a la hora de sustentar el estudio. En un trabajo realizado por Luciana Lago y otros (2020) en el que indagaron las opiniones de los jóvenes en tiempos de pandemia, se pone en evidencia la falta de cobertura del Estado en la provisión de internet y dispositivos, que generó (o agrandó) una nueva brecha que pone al descubierto la cruda desigualdad del sistema educativo argentino.

Más allá del valor del relevamiento cuantitativo estadístico, cuando decimos censar es sinónimo de conversar. Es necesario, por tanto, generar un gran conversatorio entre docentes, familias y jóvenes en el que se hable de lo vivido, se objetive la experiencia educativa y sobre todo se legitime y pondere.

Como antes decíamos, el problema del encuentro de los saberes escolares y cotidianos es de larga data y ha dado lugar a numerosos planteos y posturas. De este campo, recreamos, para el análisis que efectuamos, la visión sociocultural que considera, cuando se discute acerca del cambio conceptual, que este proceso no acontece, sino que el sujeto reconoce la actuación válida para cada contexto, sin acontecer transferencias posible entre el cotidiano y el escolar.

Esta posición teórica ha interpelado al sistema educativo mediante sucesivas investigaciones que han demostrado que efectivamente los niños de clases marginales tienen un 10 en la vida y un cero en la escuela (Carraher, Carraher y Schliemann, 1999), y entonces activan estrategias para solo un contexto, el 
cotidiano, y en el otro fracasan. Si no censamos, cada contexto seguirá su trayectoria, con nuevos episodios de fracaso escolar; no podemos perder esta oportunidad.

Para el problema de origen, esta mirada permitiría visualizar esas estrategias de resolución de problemas que los estudiantes adoptaron, provenientes de sus entornos cotidianos, a la hora de abordar las consignas escolares. Las referencias empíricas de la actividad de aprendizaje estarían dadas entonces en esos otros valores construidos fuera de la currícula escolar pero que a la vez representaron la base o el sustento para dar cumplimiento a las tareas. En cuanto a la suposición de la autoría de terceros en la producción de sus trabajos, el censo debiera relevar la producción intelectual en contextos donde efectivamente acontece la asistencia de otros, el acompañamiento, el trabajo en grupos, ya que la vida cotidiana se constituye justamente en torno a esa experiencia. En lugar de censarla como transgresión, debiera concebirse como nota constitutiva.

\section{La tentación de focalizar}

La vuelta a clase conlleva expectativas respecto de cuánto se aprendió, que se conjugan con definiciones previas de parte de los docentes respecto a los cumplimientos dados en la entrega de producciones durante la enseñanza mediada, entre otras cosas. En ellas, surge la de definir posibles agrupamientos al regreso escolar según estos niveles alcanzados.

Hemos expresado nuestra preocupación por las consecuencias de esta posibilidad, porque creemos que podrían producirse sesgos en la selección, en tanto que no habría suficientes elementos para una clara delimitación, por ejemplo. Pero más allá de eso, interesa salir de este tiempo experiencial y recordar que en nuestro país y región se ha avanzado notoriamente en los principios y leyes que rigen la inclusión educativa no solo respecto de la discapacidad sino sobre todo en cuanto a la singularidad de los sujetos.

Uno de ellos es el de promover la presencia igualitaria de todos los estudiantes en la misma sala de clase y de manera simultánea mediando algunas configuraciones de apoyo si se tratara de la discapacidad. Estos recursos servirían no solo para el grupo en su totalidad, es decir, cuando se adecúa un material de estudio para unos, sirve para todos. Focalizar podría generar un retroceso con altas posibilidades de estigmatización en nombre de un criterio operativo. Y, además, está claramente demostrado que los estudiantes más avanzados acompañan positivamente a los estudiantes con dificultades, lo que también les reporta aprendizajes (Doise, Mugny y Perret Clermont en Carretero, 1998).

La evaluación intervendría en este escenario para validar esas actuaciones, es decir, para ponderar esa actuación colaborativa y fraterna de unos estudiantes a otras. Lejos de concebirlo como un refuerzo, estamos asignando significación a un modo de ser que en la sociedad ha de adquirir alto valor, y con ello, dando sitio a la motivación interna y externa.

\section{Otros contextos, otras herramientas}

En un valioso informe sobre el estudio de los jóvenes en contextos de pandemia, Lago y otros (2020) referencian el uso sobresaliente de la aplicación WhatsApp a la hora de compartir textos o trabajos. Este uso se circunscribe a los estudiantes, aunque no a los docentes y se sustenta básicamente en el acceso que posibilita, por la presencia estable en su vida cotidiana, familiaridad de funcionamiento y menor costo.

Algunos directivos han tomado en forma gradual esta tecnología para la comunicación con las familias, sobre todo las más vulnerables, porque no cuentan con conexión por wifi en sus hogares, mediante mensajes escritos. Hemos instado a hacerlo por videollamada, dado que intervienen los rostros, y ello ayuda.

Más allá de la experiencia, tan valiosa, por cierto, este hecho activa una vez más el debate acerca del carácter transformador de la herramienta en el sentido de la mejora de la vida humana. Carina Lion (2006), en tal sentido, se pregunta si la inscripción omnipresente de las tecnologías en los tiempos actuales cambia la cognición humana como ha acontecido en otros tiempos de la humanidad con la imprenta o la escritura. Si trasladamos este interrogante a la situación que se analiza, el uso de aquella tecnología genera nuevos comportamientos en la interacción humana, porque la hace inmediata, espontánea, contingente y por tanto efectiva. La contingencia es vital en tiempos en que el ser humano necesita de respuestas de otros para su bienestar emocional, tal como lo dice Español (2010) cuando se refiere a la subjetividad infantil en la que resulta muy importante la respuesta inmediata del adulto cuidador. Por tanto, la cognición cambia porque cambia la interacción.

Resulta necesario pensar entonces el uso de tecnologías que ayuden no a la respuesta tan inmediata, pero sí no tan diferida como lo son las que resultan de las plataformas virtuales, en tiempos en que necesitamos interactuar. Los docentes podrían entonces usar esta u otra tecnología que posibilite acercarse de manera contingente a los jóvenes, porque es mucho lo que representa para ellos, configurando su uso con reglas claras de carácter funcional. Si ello sucediera, diremos entonces que el hombre cambia las tecnologías porque las adapta a su intencionalidad.

Por lo demás, Iturrioz (2019) alude al uso de las tecnologías como la expuesta como un modo de generar inclusión efectiva a la hora de la evaluación virtual, en la medida en que la herramienta sea cálida para los estudiantes. 


\section{La gente reclama notas}

La sorpresiva desaparición de la calificación numérica o conceptual irrumpió como noticia de impacto en la comunidad educativa, acostumbrada a culminar con ellas las trayectorias escolares, por efecto de los términos de la Res. CFE 363/20. Repasemos algunos de sus párrafos:

a- La aplicación de variados recursos pedagógicos que permitan indagar, sistematizar y retroalimentar los procesos de enseñanza y acompañamientos en curso. Estos recursos darán sustento a un proceso de evaluación formativa amplia e integral, que ofrezca a los/as estudiantes y las familias lecturas compartidas sobre lo realizado en esa etapa.

b- La finalidad de la evaluación de las actividades educativas durante la continuidad pedagógica es orientar mejor el proceso de enseñanza y aprendizaje, así como también promover procesos autoevaluativos en los y las alumnos y alumnas que permitan seguir aprendiendo.

c- La evaluación formativa no incluye el uso de escalas numéricas o conceptuales de calificación. Esta convención del sistema, en esta etapa, no garantiza una valoración justa y transparente de la heterogeneidad de trayectorias individuales y colectivas de las poblaciones escolares de todos los sistemas jurisdiccionales y no constituye una herramienta adecuada para orientar el proceso de enseñanza y aprendizaje.

d- Es importante distinguir el acompañamiento y seguimiento del aprendizaje de los y las alumnos y alumnas durante el período de la cuarentena que puedan estar realizando docentes y escuelas (incluso cuando involucra corrección y devolución de actividades escolares), de las instancias de evaluación que puedan ser consideradas para la acreditación de aprendizajes y la promoción de las secciones/l grado/año escolar.

Importa traer textualmente estos conceptos por la entrañable claridad respecto de la evaluación. El mismo asigna la realización de evaluaciones formativas para el tiempo de continuidad pedagógica y las delimita de la acreditación y promoción. La función que atribuye a la evaluación formativa es la de acompañar mediante devoluciones a los estudiantes y sus familias, sin calificaciones.

En las voces de docentes y familias, la falta de nota representa un problema porque los estudiantes dejaron de cumplir, tal como lo han expresado. ¿Por qué pesa tanto la nota en nuestras vidas, aún en situaciones críticas como las que estamos viviendo? ¿Por qué la insistencia, siendo evidente que habría inequidad si se asignan porque muchas familias no acceden efectivamente a las ofertas de la continuidad pedagógica? ¿Por qué nos cuesta tanto? Se trata de concepciones socioculturalmente arraigadas que sustentan esta interpretación como también efecto de los sistemas sociales meritocráticos que basan el éxito en cifras. Basta con mirar que en fútbol se gana con goles y en elecciones con votos, más allá de las apreciaciones cualitativas que podamos hacer de los jugadores y candidatos. Es menester, y será parte del legado, que muchos esfuerzos conjuntos gestemos un espacio en el que debatir estas visiones, sobre todo si generan inequidad, porque se trata nada más y nada menos que de abrir la puerta al fracaso escolar.

Por otro lado, como hemos explicado, la evaluación formativa no deja fuera la nota. Como explica Camilloni (2018) la evaluación formativa ha sido concebida para efectuar devoluciones de los trabajos de los estudiantes durante el proceso de aprendizaje, sin poner calificación mientras ello suceda. Por eso, de lo que se trata de pensar es en la corrección, en el lenguaje que empleamos y su impacto en los jóvenes y el tiempo de entrega; la autora considera que debe ser inmediato a fin de generar valor y a nuestro juicio, contingencia.

Emerge otro aspecto de la evaluación que es la retroalimentación, que representa el núcleo de la concepción formativa y que se expresa en las devoluciones y demandas de nuevas entregas, hasta alcanzar una producción que sea valorada referencialmente desde los criterios de evaluación. Y allí sí, si los sistemas lo indican, culminará con nota o concepto, pero cobijado en un proceso de ida y vuelta en el que primó de manera subyacente una visión de proceso y un sentimiento de respeto mutuo. No se trata, pues, de la nota como un problema en sí mismo, cómo llegamos a ella, cuáles narrativas la sostienen.

Surge aquí el problema de lo masivo y su relación con la posibilidad, cuando los docentes expresan que es altamente problemático efectuar devoluciones en grupos numerosos, a lo que se suman inadecuadas condiciones de trabajo docente. Nos recuerda un viejo debate que Edith Litwin (2009) protagonizó cuando se discutía la tensión masividad-calidad, a lo que le agregamos la cuota de posibilidad. En aquella opinión, la autora afirmaba que se trataba de un falso dilema en tanto que los profesores enseñan a pequeños grupos o grandes con la misma rigurosidad y que en última instancia, son otros los indicadores de calidad.

En los últimos tiempos, a nuestro juicio, se ha sobrevalorado el uso de rúbricas como instrumento para reducir aquel problema de la sobrecarga de trabajo docente. Las rúbricas posibilitan combinar criterios de evaluación con valoraciones expresadas en adjetivos, en las que se vuelcan los resultados obtenidos con marcas, cruces u otras variantes. Hemos dicho que esta propuesta no resuelve la sobrecarga de trabajo docente, y como Litwin (2009), los problemas del trabajo docente han de resolverse de otro modo y no renunciando a efectuar devoluciones a nuestros estudiantes. Por lo demás, cuando se desvirtúan las rúbricas, se termina en prácticas de otros tiempos, de tipo medible, observable y tangible. Nada más lejano a sus intenciones. 
Hemos contrapropuesto que una posibilidad es efectuar devoluciones en dos planos; en el primero, partiendo de los criterios de evaluación, adjetivar distinto para cada estudiante, lo que significa que se escribirán adjetivos diferentes para cada estudiante, breves pero consistentes. De esta manera, las tendencias homogeneizadoras que se ven en el uso de las rúbricas, serán superadas porque habrá un adjetivo diferente para cada trabajo. En el segundo, establecer niveles de avance, que permitan registrar las distintas versiones de la producción del estudiante; ello posibilitará que se visualicen los resultados obtenidos como parte de un proceso.

Respecto de esto último, Vygostky (1979) fue uno de los primeros críticos de las pruebas cuantitativas homogeneizadoras, que en su tiempo fue a las pruebas de Cl. Según él, la capacidad cognitiva no es una entidad natural sino un constructo sociocultural que surge de la interacción del niño con su entorno. Por lo tanto, el sujeto de la capacidad no es el niño aislado sino el par niño-cultura como aptitud decreciente para aplicar nuevos instrumentos culturales a los propios procesos psicológicos.

El concepto de evaluación apareció en el contexto del concepto Zona de Desarrollo Próximo, que la compromete no solo a identificar los procesos cognitivos del niño ya desarrollados sino los que se encuentran en proceso de desarrollo. Ello depende de una interacción cooperadora entre el niño y el adulto que representa la cultura, que le ayuda a adquirir los instrumentos simbólicos necesarios para el aprendizaje. Por lo tanto, las funciones cognitivas emergentes se pueden identificar incorporando el aprendizaje en cooperación en el procedimiento de evaluación. Cuantitativamente la Zona de Desarrollo Próximo es una medida de la diferencia de rendimiento con y sin ayuda.

Resulta muy clara la visión del célebre pensador a la hora de pensar en los diferentes niveles de progreso en la producción de los trabajos de los estudiantes. Las funciones intelectuales emergerán en la medida en que haya un proceso asistido con momentos diferentes que permitan ver tal emergencia.

Para terminar, el uso del portafolio colabora en la apuesta a lo diverso y procesual. Como ya sabemos, consiste en que el estudiante elige y distingue sus mejores trabajos y los dispone en una carpeta. Para ello, hemos dicho que la labor docente será la de orientar mediante criterios de evaluación referidos a los saberes que se ponen en juego como también a otras habilidades personales que se desprenden de este recurso. De este modo, le hacemos saber lo que importa en términos de conocimiento a la vez que promovemos la autorregulación de los aprendizajes, que es otro componente intrínseco de la retroalimentación formativa. Ello compromete a la devolución sin duda alguna, y volveríamos al problema de la masividad. Hemos dicho al respecto que ellas pueden hacerse mediante contactos personales, en tanto que el uso del recurso lo favorece. En caso de que suponga administraciones diferentes de tiempo al convencional, habrá que hacerlo. Parte del legado será, sin duda alguna, la generación de espacios y tiempos diferentes al clásico formato escolar.

En tiempos de continuidad pedagógica, la familia ha de ser una fuente importante a la hora de obtener referencias respecto de las experiencias de sus hijos. Hemos de concebirlo como un interlocutor válido sin duda alguna, y será parte de los legados.

\section{De criterios e instrumentos}

Será mayor el desarrollo en este punto en tanto entendemos que representa el núcleo de toda práctica evaluativa.

\section{- De los criterios}

Citamos algunos componentes que entendemos constitutivos:

1. Los criterios de evaluación son parámetros de referencia conceptuales que evidencian el valor, la significación que se le asigna a algo. Para el caso de los aprendizajes escolares, estos criterios se expresan mediante actos de pensamiento que se espera que los estudiantes ejerzan sobre el conocimiento. Dicho, en otros términos, se espera que el estudiante reflexione, conceptualice, reconozca, exprese un juicio crítico respecto de un determinado contenido escolar en el marco de una actividad evaluativa. Son parámetros porque esperamos que sucedan, como referencia de aprendizaje. Como si fueran fotografías de una fiesta en la que seleccionamos las mejores escenas para retratarlas como una experiencia agradable.

2. Concebimos la construcción de los criterios de evaluación en términos de actos de pensamiento desde el valor que asignamos al pensamiento en el aprendizaje. El tiempo actual, de irrupción formidable de información mediante las redes, requiere formar un sujeto crítico, que pueda reconocer, distinguir y formar un juicio propio, que pueda apreciar cualidades más allá de lo que se le imponga como realidad. Es el lugar de la autonomía y del proyecto propio.

3. Cuando evaluamos mediante criterios, la labor docente es adjetivar, es decir, frente a la producción lograda y el criterio como referencia, se tratará de expresar valoraciones que indiquen los resultados obtenidos, parciales o totales según si se trabaja con niveles de progreso o no.

4. Los criterios comprometen a ponderar, esto es, decir acerca de la jerarquía de importancia de cada contenido, que puede expresarse en el orden en que se presentan o con puntuaciones si se trata de una instancia de acreditación. Nuevamente, el punto no es la nota en sí sino lo que hacemos con ella.

5. Mucho se ha dicho respecto de compartir los criterios. Creemos que es una práctica valiosa no solo en nombre de la transparencia sino porque se muestran los nudos centrales de una asignatura o disciplina. Decíamos antes que la evaluación es legitimadora; creemos que es estratégico 
mostrar los conocimientos que son importantes en la materia y con ello legitimar, lo que resulta importante mediante los criterios, porque es formativo.

6. Los criterios de evaluación no se cristalizan, sino que cambian. No solo porque pretendemos inscribirlos en evaluaciones auténticas (y la realidad es cambiante) sino porque además como producto del censo de entornos, en el marco de la continuidad pedagógica, se apreciará la emergencia de saberes novedosos tales como las competencias digitales, la organización personal, el procesamiento de información en pantallas digitales, entre otros.

7. Importa también decir que los criterios de evaluación, en caso de favorecerse en las consignas la autorregulación, tanto en términos de revisar errores y aciertos como también de reconocer formas superadoras, deben ponderarse fuertemente, esto es, incluirlas como criterio y jerarquizarlas. De otro modo, podrían quedar en la dimensión experiencial, lejano a la potencialidad que conlleva.

8. Los criterios de evaluación no incluyen los requisitos de tiempo y forma que a veces se piden en los trabajos escritos, sino cuando esos aspectos tengan intencionalidad pedagógica. Así, los tiempos en la evaluación formativa son relativos, lo cual no quiere decir que se acepte cualquier condición, sino que se personalizan en la medida en que los niveles de progreso son diferentes. Respecto de la forma, para el caso de la escritura, es importante que se incluya si se concibe en su sentido epistémico, es decir, si la buena escritura sirve para aprender; en cambio, si asume un valor instrumental separado de la producción en sí, no debe ponderarse o hacerlo en su justa dimensión. Es importante considerar si ha existido alguna instancia formal de enseñanza de la escritura cuando es superior o académica porque se trata de un proceso de alta complejidad lejano a la adquisición espontánea (Vygotski, 1997). De igual modo, es importante ponderar el valor de la escritura según sea la asignatura, ya que no en todas adquiere la misma significación.

9. Desde la perspectiva sociocultural que hemos referenciado en este trabajo, el pensamiento no se ejerce de manera solista. Wertsch (1999), al respecto, efectúa una crítica a los enfoques psicológicos que interpretan la acción humana desde la dimensión individual y considera que los individuos operan con modos de mediación con los que construye una relación dialéctica. Dispone un ejemplo de un deportista de salto en alto cuyos logros dependen de sus destrezas a la par de la ayuda de una garrocha. De igual modo, plantea un ejemplo de aprendizaje escolar matemática a la que define como mediación semiótica, como es la resolución de una operación de multiplicación. El autor compara la forma de resolver una operación simple por cálculo mental que no requiere de recursos ajenas al mismo, con una multiplicación de 3 cifras, ante la que el cálculo mental no alcanzaría, por lo que usuaríamos una calculadora. Mientras que la primera acción es individual y mental, la segunda es con mediación semiótica (hay implicada una herramienta cultural), por lo que se trataría del sujeto con la herramienta, o acción mediada. Entendemos que los criterios de evaluación han de ponderar el uso de estos recursos que pueden ser materiales o semióticos pero que en definitiva son constitutivos de la resolución de problemas en las que media el pensamiento.

\section{- De los instrumentos}

Recogemos información de los aprendizajes de nuestros estudiantes a través de los instrumentos de evaluación, esto es, exámenes escritos, orales, experiencias prácticas, representaciones gráficas, entre otros.

Desde una actitud analítica, reconocemos la importante influencia que generan en las respuestas de los estudiantes al momento de ser examinados. El tipo de consignas que se plantean, la claridad en su formulación, los criterios de evaluación en que se apoyan, las condiciones de realización en el aula, nuestra propia actitud docente al momento de presentarlas, inciden de manera esencial en el rendimiento.

La necesaria revisión de los instrumentos de evaluación requiere revisar las dificultades de comprensión que generan las consignas; en términos de la teoría sociocultural, aquellas consignas de evaluación que suscitan enormes distancias con respecto a las experiencias vitales de los estudiantes. Estamos ante un problema complejo, y por ello nos detendremos un poco en este punto.

Michael Cole (1999), destacado referente en la denominada "Psicología cultural", expresa la importancia que asume, al momento de estudiar la resolución de problemas de las personas, conocer sus patrones culturales genuinos, que deviene del análisis cotidiano de sus vidas. En el marco de importantes investigaciones transculturales, esta corriente de pensamiento sostiene las personas desarrollan herramientas culturales y destrezas cognitivas asociadas a los dominios de la vida en que estas herramientas son importantes. De esta manera, las diferencias culturales en los procesos cognitivos entre diferentes grupos sociales (escolarizados en sociedades occidentales y no escolarizados en sociedades tribales) radica no en la existencia de procesos cognitivos en un grupo e inexistencia en otro, sino en las tareas experimentales y las conclusiones que se extraen de ellas.

Expresa este pensador:

En 1963 me pidieron que asesorara sobre un proyecto para mejorar el rendimiento educativo de los niños que vivían en Liberia, el país de África Occidental (...) La lista de dificultades que poseían los niños tribales era larga. Tenían problemas para distinguir entre diferentes formas geométricas porque, según se decía, experimentaban graves problemas perceptivos. También se decía que no 
sabían cómo clasificar y que, cuando tenían que elegir entre pensar y recordar recurrían, al recuerdo repetitivo...

Las visitas a las escuelas me mostraron la base para algunas de estas afirmaciones. En muchas aulas vi a los estudiantes ocupados en tareas de recuerdo repetitivo. En encuentros con los habitantes del lugar que habían organizado su vida en función de las prácticas culturales indígenas y no del sector económico moderno en cuyo apoyo se había introducido la escolarización, obtuve una impresión completamente diferente de las destrezas intelectuales de los kpelle. A juzgar por la manera en que componen rompecabezas o estudian matemáticas en la escuela los kpelle parecían tontos; a juzgar por cómo se comportaban en los mercados, en los taxis y en muchos otros entornos, parecían inteligentes. ¿Cómo podían ser tontos e inteligentes al mismo tiempo? Necesitábamos estudiar las actividades cotidianas de las personas que implicaban medir, estimar, contar, calcular, como condición previa para diagnosticar la comprensión matemática indígena en relación a la escolarización (Cole, 1999, s/p).

Del censo de entornos, como antes decíamos, reconocemos el aprendizaje de diversas nuevas habilidades. Para el enfoque expuesto, se trataría entonces de incluirlas y ponderarlas mediante criterios de evaluación y consignas expresas. A modo de ejemplo, las competencias digitales aprendidas o la resolución estratégica de problemas y obstáculos que hayan encontrado. De igual modo, la integración de mediaciones sociales y experiencias de autorregulación

A modo de síntesis, enumeramos algunas características que debieran reunir los instrumentos de evaluación:

1. validez de contenido (Camilloni y otras, 1998): compromete la pregunta referida a qué vamos a incluir en la prueba, dado que es imposible tomar todo lo que se enseña. Para resolver este intrincado problema, debemos tener claridad primero acerca del universo, es decir, de todo lo que enseñamos cuando enseñamos. Sabido es que en el aula se enseñan más cosas respecto del proyecto original. Una manera apropiada es enumerar cotidianamente las actividades de aprendizaje que efectuamos en el aula, de lo cual derivaremos la frecuencia de unas sobre otras. Por tanto, todo lo que enseñamos en el aula es el universo, y aquello que enseñamos con mayor frecuencia es la muestra. Ello será entonces lo que integrará el contenido central de los instrumentos evaluativos;

2. atender a las características de las consignas en términos de evitar que sean solamente dirigidas a reconocer los saberes escolares; ello no significa que toda experiencia debe ser incluida, sino que se abra el juego para que el estudiante pueda integrar saberes que, en contextos de continuidad pedagógica, consideró valioso aprender;

3. en el sentido de lo anterior, asignar un lugar específico para valorar las competencias digitales. Como dicen algunos, el entorno de los jóvenes es el celular. Reconocer el enorme repertorio de habilidades con que cuentan y las potencialidades que conllevan es un modo de ejercer validez ecológica;

4. diversificar los formatos de consignas, incluyendo casos, problemas, dibujos, historias, es decir, modos de representación diversos. En los tiempos que corren, la producción de "memes" representan de manera clara el sentir popular, que para Jerome Bruner (1991) dan cuenta de la narrativa popular;

5. invitar a relatar problemas y aciertos que hayan vivido en el tiempo de distanciamiento escolar, para el contexto de continuidad pedagógica. Se relaciona esta iniciativa con el valor que tiene la consideración del contexto a la hora de considerar la actuación del sujeto;

6. solicitar que se describa el método usado para estudiar, en términos de favorecer la autorregulación;

7. incluir el lugar de la ayuda social, esto es, en los términos vygotskianos antes expresados, documentar la emergencia de nuevas funciones psicológicas a partir del sostén de otras personas que asistan en la resolución de problemas;

\section{Lo que queda}

Como decíamos en un inicio, lo expresado en el trabajo representa un espacio de múltiples conversaciones con docentes y directivos de escuelas primarias y secundarias que hacen llegar sus voces y demandas. Hemos procurado acompañarlos con reflexiones y propuestas, con un pié en la continuidad pedagógica, pero con una mirada retrospectiva y prospectiva analítica hacia las prácticas evaluativas escolares.

En términos de las normativas que generan el movimiento hacia el encuentro, importa remarcar la potencialidad de la reflexividad docente a la hora de situarse críticamente frente a prescripciones que interpelan sus prácticas y requerir del análisis compartido. Aún cuando resulte difícil pensar movimientos efectivos hacia la mejora de las prácticas evaluativas, la actitud resistente y reflexiva es un paso decisivo hacia ella. En torno a lo que nos ocupó en este espacio, la posibilidad de mirar otras referencias empíricas a la hora de evaluar que contemplen integralmente la actuación de niños y jóvenes, es un signo destacado.

Sin más, la expectativa es que la inscripción de muchos componentes del legado que se construirá en contextos de continuidad pedagógica, en el currículum escolar. Porque detrás de toda crisis, hay una oportunidad. Y en esta vemos la de escribir otro capítulo para la evaluación. 


\section{Referencias bibliográficas}

Baquero, R. (1997). Vygostky y el aprendizaje escolar. Buenos Aires: Aique.

Bruner, J. (1991). Actos de significado. Más allá de la revolución cognitiva. Madrid: Morata.

Camilloni, A. (2015). La responsabilidad social y pedagógica de la evaluación de los aprendizajes. En: Revista Itinerarios educativos pp.8-8

Camilloni, A. (2018). Conferencia "La evaluación, la enseñanza y el aprendizaje". En: Jornadas Formar FADU. Universidad de Buenos Aires.

Carraher, T.; Carraher, D., Schliemann, A. (1999). En la vida diez, en la escuela cero. Buenos Aires: Siglo XXI.

Camilloni, A., et. al. (1998). La evaluación de los debates didácticos contemporáneos. Buenos Aires: Paidós.

Carretero, M. (1998). Desarrollo y aprendizaje. Buenos Aires: Aique.

Cole, M. (1999). Psicología cultural. Madrid: Morata.

Consejo Federal de Educación de la República Argentina (2020). Resolución CFE 363/20. Buenos Aires.

Diaz Barriga, F. (2005). Aprendizaje basado en problemas. De la teoría a la práctica. México: Trillas: Carlos Sosa Ayape.

Español, S. (2010). El desarrollo como estrategia adaptativa. Características específicas de la especie humana. En Revista de Psicología. N¹1. Facultad de Humanidades. Universidad Nacional de La Plata.

Iturrioz G. (2019). Entradas para pensar la evaluación de los aprendizajes universitarios desde una perspectiva inclusiva. Trabajo final de Especialización en Tecnología educativa. Facultad de Filosofía y Letras. Universidad de Buenos Aires. CABA.

Lago L., et. al. (2020). Informe "Estudiar en tiempos de pandemia, voces y experiencias de estudiantes". IESyPPat. UNPSJB. Comodoro Rivadavia.

Lion C. (2006). Imaginar con tecnologías. Relaciones entre tecnologías y conocimiento. Buenos Aires: Ediciones La Crujía.

Vygostki L. (1979). El desarrollo de los procesos psicológicos superiores. Barcelona: Grijalbo.

Vygostki L. (1997). Obras escogidas Tomo II. Pensamiento y lenguaje. Barcelona: Visor.

Wertsch J. (1999). La mente en acción. Buenos Aires: Aique. 